\title{
The features of the through-hole by picosecond pulse laser helical drilling on alloy
}

\author{
D. Q. Yuan*,J.T.Xu \\ School of science, Huai Hai Institute of Technology, Lianyungang, Jiangsu 222005, China \\ cumtydq@163.com
}

\begin{abstract}
Keywords: Picosecond laser, helical,through hole,repetition rate, multi-scale;
Abstract. Picosecond laser was employed to drill through holes on alloy of GH2132 by helical technique.The effects of repetition rate and processing time on the qualities of through holes were investigated.Morphology of holes of irradiated areas were analyzed by scanning electron microscope(SEM). The results indicated the repetition rate had major effection on through hole quality and machining efficiency.With the repetition rate of $100 \mathrm{kHz}$, the multi-scale through holes could be machined.It was demonstrated that the entrance ends and the exit ends of the through holes were quite smooth where without accumulation of spattering material and formation of a recast layer and microcracks. Comparing the entrance and exit diameters of through holes, no conicity phenomenon was observed. All in all,picosecond laser helical drilling with optimal parameters could be an effective technique for manufacturing through holes with very high quality.
\end{abstract}

\section{Introduction}

At present, laser manufacturing has been used as an effective technology for machining in various materials, such as metal, silicon and ceramic ${ }^{[1]}$.With the development in aeronautical manufacturing technology and the continuous improvements of the conversion efficiency require the engines and blades to have a better machine performance ,longer life, and a higher precision ${ }^{[2]}$. In order to realize those purposes, it is important to drill micro-holes in this material for some special applications ${ }^{[3]}$. Until now, the processed holes which with high-efficiency and high-quality still face enormous challenges.Laser drilling as a versatile and reliable technology has been applied in the aerospace industry to produces shaped film holes ${ }^{[4]}$. For this purpose,more researches have been focused on ultra-short pulsed laser,typically femtosecond and picosecond laser, and it was showed that they had many advantages such as minimal damages, high precision and efficiency. The great demands in laser drilling had promoted the development of laser machining technology from single-pulse drilling to percussion drilling ${ }^{[5]}$.Kamlage et al. ${ }^{[6]}$ demonstrated the results of investigations on drilling metals by femtosecond laser pulses, and it was noticed that the generation of laser-induced metal vapor and small metal particles (debris), trans-formation of laser pulses into light filaments, and low-fluence finishing were important to the formation of the high quality holes. And then, Bruneau et al. ${ }^{[7]}$ studied the mechanisms of ultra-fast laser ablation during deep-drilling of metals using plasma diagnostics, and it was showed that the nanoparticles were important on the accuracy of micro-machining. One of the most efficient and accurate drilling technologies namely helical drilling was reported ${ }^{[8]}$.It was reported that a combination of picosecond laser with an optimal parameter setup and helical drilling method has been proposed to gain microholes with different shapes on stainless steel. Precision drilling with picosecond laser maybe a better method to obtain the high-quality holes with the optimal adjustment of parameters such as average power,repetition rate, and focal position ${ }^{[9]}$.

Although there are many studies ${ }^{[10,11]}$ demonstrate the helical drilling was a good choice for machining microholes on the workpiece, however the detail research about the evolution of the hole by picosecond laser drilling was absent. Especially, the influence of the repetition rate on the formation of the through holes has not been investigated thoroughly. This paper mainly focuses on the mechanisms of picosecond laser helical drilling,and discussion two significant processing factors on the hole quality, which including laser repetition rate and the machining time. 


\section{Experimental setup}

In order to minimize the heat affect zone(HAZ) around the kerf and holes on the workpiece of alloy material GH2132(with a thickness of $500 \mu \mathrm{m}$ ), ultra-short pulses laser with pulse $15 \mathrm{ps}$ at $532 \mathrm{~nm}$ wavelenght is applied.The output laser power is set at $6 \mathrm{~W}$ and the repetition rate could be adjusted from $100 \mathrm{kHz}$ to $1000 \mathrm{kHz}$. According to the experimental setup, we set the beam expanding ratio at 6 , the laser focus spot was about $20 \mu \mathrm{m}$, the assist gas pressure was set at $0.6 \mathrm{MPa}$. The manufacturing system which incorporated a picosecond laser into a three-axis position stage, and a high speed laser beam rotation apparatus which called helical model was introduced in order to eliminate the adverse effect which caused by the discrepancy of laser intensity distribution within the spot and the recast influence during laser drilling. The experimental setup had been introduced in detail ${ }^{[12]}$.

\section{Results and discussion}

Through this experiment, we would obtain the suitable value of pulse repetition rate and the laser machining time for the further experiment. Through the pre-test, we set the laser power at $6 \mathrm{~W}$ and the helical speed at $60 \mathrm{r} / \mathrm{s}$.
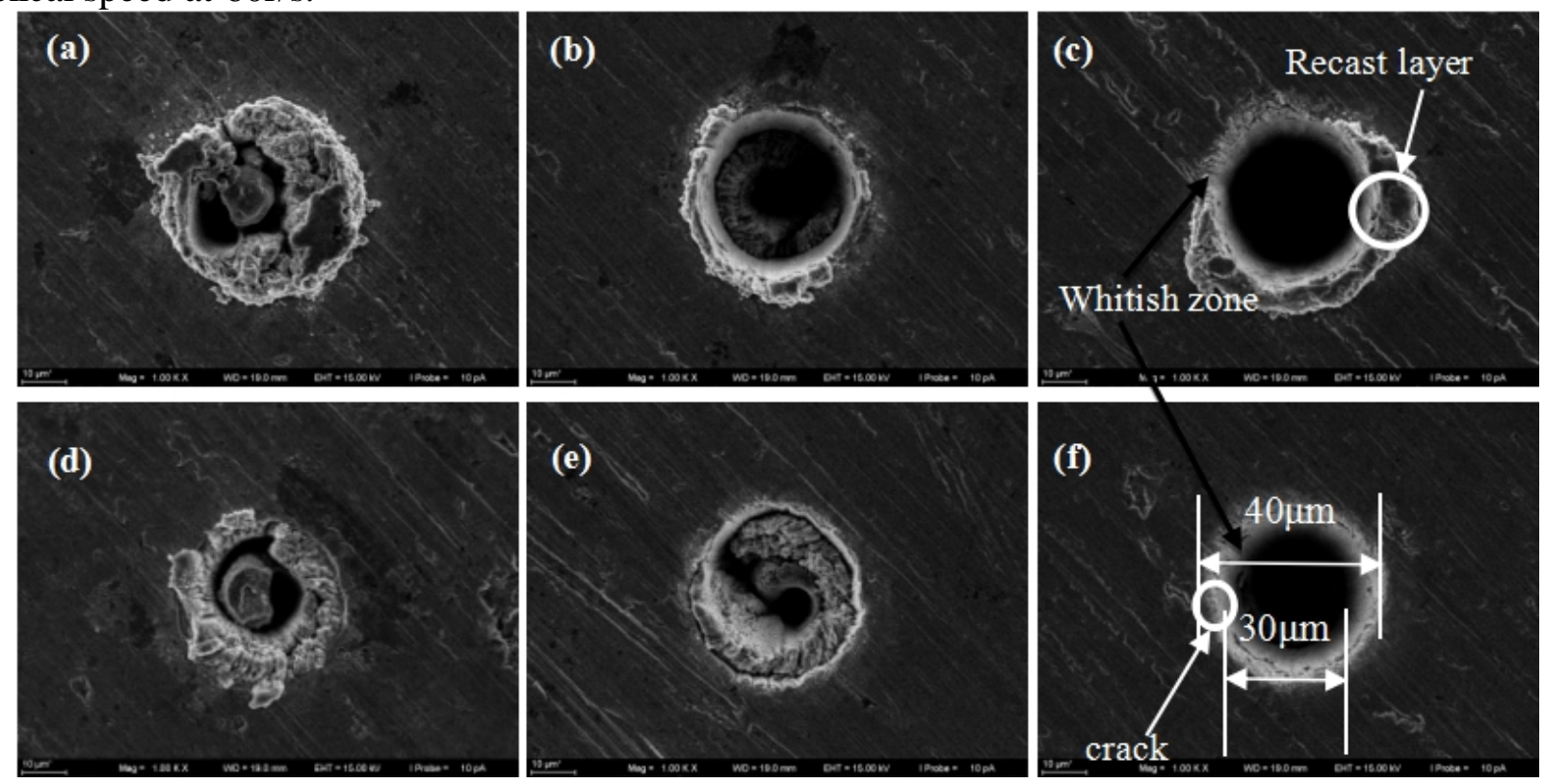

Fig.1.SEM micrographs showing the entrance end of the holes with different Repetition rate and processing time(laser power of $6 \mathrm{~W}$ ) .(a) $200 \mathrm{KHz}, 100 \mathrm{~ms}$ (b) $200 \mathrm{KHz}, 500 \mathrm{~ms}$ (c) $200 \mathrm{KHz}, 1000 \mathrm{~ms}$ (d) $500 \mathrm{KHz}, 100 \mathrm{~ms}(\mathrm{e}) 500 \mathrm{KHz}, 500 \mathrm{~ms}(\mathrm{f}) 500 \mathrm{KHz}, 1000 \mathrm{~ms}$;

As shown in Fig.1,the SEM images of the entrance end of the hole, drilled on the alloy material GH2132 without any treatment, and a series of circular surface with an intended radius of $40 \mu \mathrm{m}$ were machined with different laser repetition rate and different processing time.Here, we set the processing time at $100 \mathrm{~ms}, 500 \mathrm{~ms}$ and $1000 \mathrm{~ms}$, respectively. The unremoved material in the circle area became smaller with increasing processing time from $100 \mathrm{~ms}$ to $1000 \mathrm{~ms}$. When compare the results of Fig.1(a) and (d), Fig.1(b) and Fig.1(e), we can find the degree of the material removed were obviously different. At $200 \mathrm{kHz}$ and $500 \mathrm{kHz}$, when the laser power is $6 \mathrm{~W}$, which means the fluences were set at $0.6 \times$ $10^{-2} \mathrm{~J} / \mathrm{mm}^{2}$ and $0.24 \times 10^{-2} \mathrm{~J} / \mathrm{mm}^{2}$. As shown in Fig. 1(c) and Fig.1(f), the diameter of the periphery of the hole was about $40 \mu \mathrm{m}$, and the inner diameter was about $30 \mu \mathrm{m}$. In addition, the middle area was a

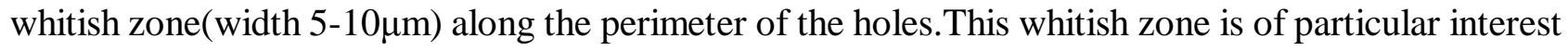
as potential recast layer that re-solidifies on the side wall of the holes during laser drilling,which had been reported that the difference in elemental composition between the whitish zone and the bulk material is not significant, there is only a slight increase in the content of $\mathrm{C}$ and $\mathrm{O}^{[8]}$. When machining the through-hole using higher pulse energy,some recast layer has emerged on the side of the hole as shown in Fig.1(c), however, the machining efficiency of this condition was more higher than the lower 
pulse energy which is shown in Fig.1(f), e.g.there is some um-removed material on the taper bottom.It demonstrates that the crack had emerged on the whitish is shown in Fig.1(f), this will become the source of the defect. So, the controlling of the repetition rate and the processing time are very important for the drilling holes, an optimal parameters will make the machining efficiency and the hole quality with optimization.

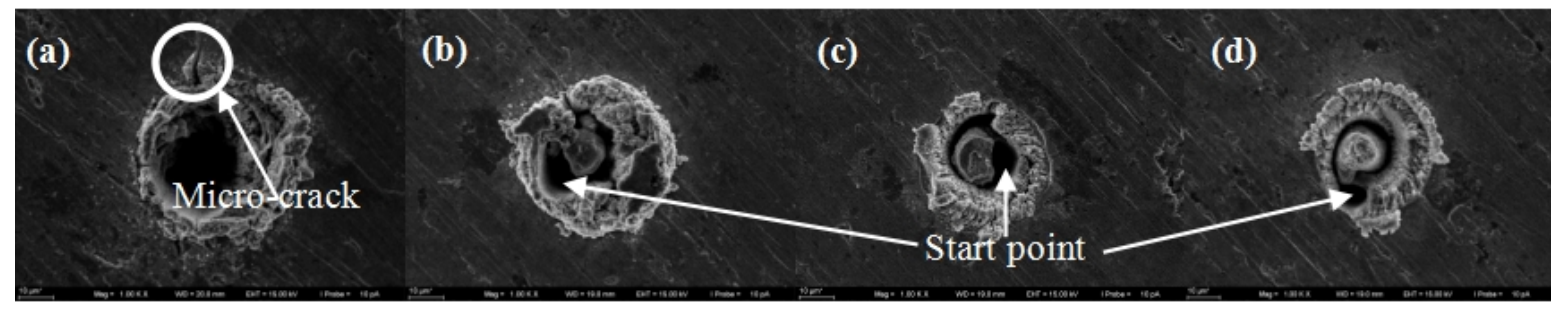

Fig.2.SEM micrographs showing the entrance end of helical drilling hole(processing time of $100 \mathrm{~ms}, 6 \mathrm{~W}, 60 \mathrm{r} / \mathrm{s}$ ) at different repetition rate(a) $100 \mathrm{KHz},(\mathrm{b}) 200 \mathrm{KHz},(\mathrm{c}) 500 \mathrm{KHz},(\mathrm{d}) 1000 \mathrm{KHz}$;

The influence of the fluence on the process efficiency was so important ${ }^{[13]}$, we investigate the influence of repetition rate on processing through-holes, and compared the results machined with different repetition rates as shown in Fig.2.It was clearly noticed that the amount of removed material was decreased with increasing the repetition rates from $100 \mathrm{kHz}$ to $1000 \mathrm{kHz}$.In Fig.2(a), the processing zone could see a whole hole, although there exist an amount of melting and spattering contents at the entrance end, although, there present some microcracks along the edge of the hole.As shown in Fig.2(b),(c) and (d), the start point was clearly observed,this was due to the processing was only one single circle.However, because at $100 \mathrm{kHz}$, the fluence was more larger,so, this could not present in the Fig.2(a).Meanwhile, with increasing the repetition rate, there exist cylindrical un-removed material on the center of the processing area.The reason for this phenomenon was the single pulse energy was decreased with increasing the repetition rate.In additional,with increasing repetition rates the so-called heat accumulation effect becomes a considerable issue ${ }^{[14]}$. With higher repetition rate, the interval time of the adjacent pulse be shorter, and there is less time for debris to escape,so,the ablated material tended to deposit on the sidewall and bottom. This is unfavorable for the formation of the through-hole.Although higher repetition rate could increase the number of pulse per second, but the pulse energy will be reduced, this will make the processing efficiency be lower.So, when keep the laser power unchanged, an optimal repetition rate is $100 \mathrm{kHz}$.
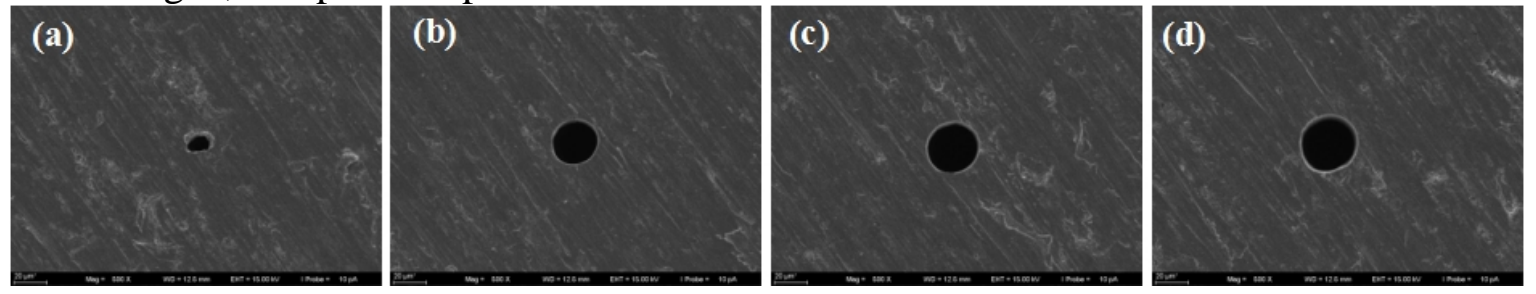

Fig.3. SEM micrographs showing the exit end of the through-holes with pulse repetition rate of $100 \mathrm{KHz}$ and different drilling time(a)100ms,(b)500ms,(c)2000ms,(d)3000ms;

The SEM images in Fig.3,show morphology of the exit end of the through-holes which drilled at $100 \mathrm{kHz}$ and with different drilling time.It was demonstrated that the exit end of the hole was very clean for different processing time.In Fig.3(a), the diameter of the exit end of the hole is about $20 \mu \mathrm{m}$, it was less than the intended diameter of $40 \mu \mathrm{m}$. With increasing the processing time to $2000 \mathrm{~ms}$, the diameter is about $40 \mu \mathrm{m}$ as shown in Fig.3(c). With continue increasing the processing time, the diameter of the exit hole will keep constant, and it is coincide to the pre-set value.Due to the experiment is equipped with the Coaxial blowing system,the molten material could be removed laterally and does not need to be expelled axially along the side wall of the hole to the entrance end as the percussion drilling ${ }^{[15]}$.So, with the optimal parameters, the higher geometrical precision of the hole could be achieved by the application of the helical drilling technology. 


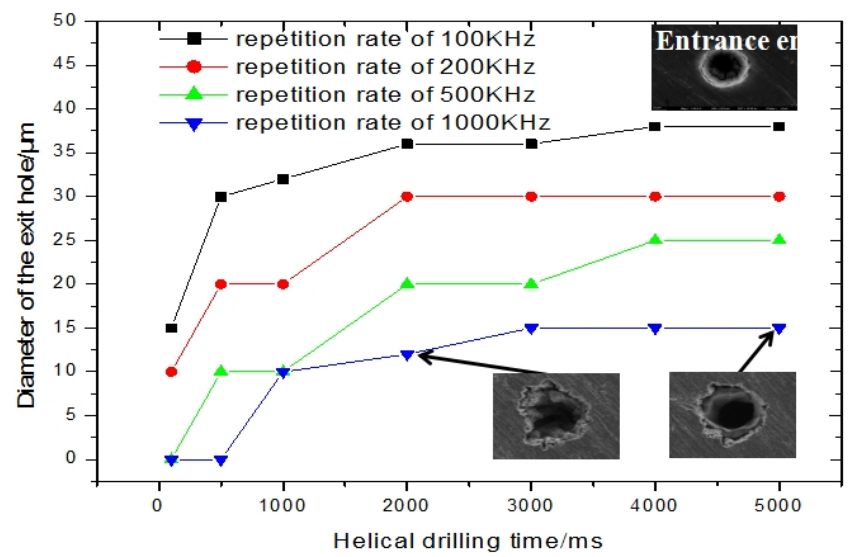

Fig.4 The diameter of the exit hole dependence on the helical drilling time and pulse repetition;

Though laser helical drilling process was complex, it was considered that high efficiency and good quality could be achieved at an optimum condition.It could be believed that the laser repetition rate and the processing time were mainly responsible for ablation efficiency and quality compare to other parameters.Fig. 4 show the relationship between the helical drilling time and the diameter of the exit hole when drilled the through-holes with different repetition rates. The measured exit holes diameter were lessthan the intended size,especially, when the laser repetition rate was $1000 \mathrm{kHz}$, the exit holes diameter is only about $15 \mu \mathrm{m}$. As shown in Fig.4, during the processing time was between $2000 \mathrm{~ms}$ and $5000 \mathrm{~ms}$, the hole diameter increased slowly.At this experiment, we set the intended diameter of $40 \mu \mathrm{m}$, however, the maximum exit end diameter were about 38-,30-,25-,15 $\mu \mathrm{m}$ respectively, when the repetition was set at 100-,200-,500-,1000kHz. It could be seen that the diameter increased significantly when the laser processing time was increased from $100 \mathrm{~ms}$ to $1000 \mathrm{~ms}$, and with an further increasing of the processing time,the change of the diameter was slowly.For the processing time of $100 \mathrm{~ms}$, the through-hole could not be fabricated when the repetition rate was $500 \mathrm{kHz}$ and $1000 \mathrm{kHz}$. With an increasing processing time to $500 \mathrm{~ms}$, using the repetition rate of $500 \mathrm{kHz}$, the through hole could be fabricated, although the exit end diameter was more less than the pretend value,this means the through-hole with a conicity. This phenomenon had appeared on the other situation, and it could be demonstrated by the difference value between the entrance end and the exit end diameters. As shown in Fig.4, when the repetition rate was $1000 \mathrm{kHz}$, the shape of the exit end of the through hole was not precision, this will led to the fail processing. This was due to the lower energy would not evaporate the material directly,then will lead to the melting material and re-solid on the bottom or on the side wall be presented, and will make the exit hole diameter become smaller.In generally, when the pulse duration is less than 10 picosends, the removal of material mechanism is depend on the evaporation, the formation recast layer and the thermal damage to the workpiece could be significantly reduced.

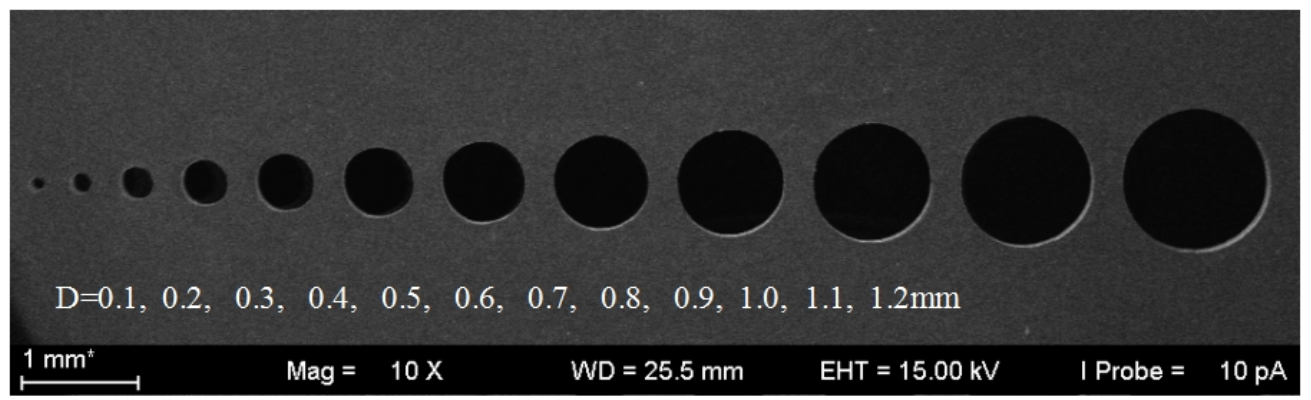

Fig.5. SEM micrographs showing the through-holes processing with pulse repetition rate of $100 \mathrm{KHz}$ and power of $6 \mathrm{~W}$, the fabricated hole with different diameter which in the range of $0.1 \mathrm{~mm}$ to $1.2 \mathrm{~mm}$.

The SEM images in Fig.5 show morphology of the through-holes with an intended diameter in the range of $100 \mu \mathrm{m}$ to $1200 \mu \mathrm{m}$ had been fabricated by picosecond helical drilling at $100 \mathrm{kHz}$ and helical speed of $60 \mathrm{r} / \mathrm{s}$, the processing time was $10 \mathrm{~s}$.Fig. 5 shows the morphology of the exit end of through-holes which with different diameters.It was demonstrated that the machined hole with high 
quality when set the optimal parameters.It means the helical method has a potential application in fabricating multi-scale and high precision through-hole.

\section{Conclusions}

In the present study, we performed a detailed research of picosecond laser helical drilling through-hole,from which the optimal drilling parameters including the repetition rate and processing time were gained,and the multi-scale diameter of through-holes could be manufactured with a higher quality such as shape accuracy and dimension accuracy. It was demonstrates that the optimal parameter for drilling through-hole was at a laser power of $6 \mathrm{~W}$, the repetition rate of $100 \mathrm{kHz}$, and the helical speed was 60r/s. The entrance end and exit end diameters of the through-hole were approximately equal to each other when using above parameters, then a higher quality hole could be fabricated. In addition, the micro-holes circularity was fairly good,and without an accumulation of spattering material and the formation of conicity phenomenon and microcracks. However, there exist some limitation about our study, that the drilling parameters could not be generalized due to the laser drilling is material dependent.And the formation mechanism of ripple structures during the drilling should do more experiment to investigate this phenomenon.

\section{Acknowledgments}

The authors would like to acknowledge the National Natural Science Foundation of China (Grant No. 51405181), the Natural Science Foundation for Youths of Jiangsu Province (Grant No.

BK20130407), the Colleges and Universities Natural Science Foundation of Jiangsu Province (Grant No. 13KJB460001).

\section{References}

[1] W. Schulz, U. Eppelt, R. Poprawe:J. Laser Appl. Vol. 25 (2013),p.199

[2] Klocke F., Klink A., Veselovac D., Aspinwall D. K., Soo S. L., Schmidt M., Schilp J., Levy G., Kruth J.-P: CIRP Annals-Manuf. Technol.Vol.63(2014), p.703

[3] Díaz, J., Rubio, L: J. Mater. Process. Technology.Vol.143-144(2003),p.342

[4] J.H.Zhang,T.C.Lee,C.L.Wu,C.Y.Tang:J.Mater.Process.Technol.Vol.123(2002),p.75

[5] R.S.Bunker:J. Heat Transfer.Vol.127(2005),p.441

[6] Kamlage, G., Bauer, T., Ostendorf, A., Chichkov, B.N: Appl. Phys. A: Mater.Vol.77(2003),p.307

[7] Bruneau, S., Hermann, J., Dumitru, G., Sentis, M., Axente, E:Appl. Surf. Sci.Vol.248(2005),p.299

[8] Hongyu Zhang,Jianke Di,Ming Zhou,Yu Yan,Rong Wang:Appl.Phys.A.Vol.119(2015),p.745

[9] X.C. Chen, L.F. Ji, Y. Bao, Y.J. Jiang:J. Eur. Ceram. Soc. Vol.32(2012),p.2203

[10] Yongsheng Liu,Chunhui Wang,Weinan Li,Litong Zhang,Xiaojun Yang,Guanghua Cheng,Qing Zhang:Journal of Materials Processing Technology.Vol.214(2014),p.3131

[11] Ying Zhang, Yuqian Wang, Junzhan Zhang,Yongsheng Liu,Xiaojun Yang,Qing Zhang:Ceramic International.2015,Vol.41(2015),p.6525

[12] D.Q.Yuan,J.K.Di,M.Zhou,J.T.Xu:Journal of russian laser research.Vol.38(2017),p.112

[13] D-Q. Yuan, J-T.Xu:Lasers in Eng. Vol.21(2011),p.365

[14] Trtica M., Gakovic B, Batani D., Desai T., Panjan P. and Radak B: Applied Surface Science.Vol.253(2006),p.2551

[15] Varro S. and Ehlotzky F:Physical Review A: Atomic, Molecular and Optical Physics.Vol.54(1996),p.3245 